\section{Manchester iStent study: 3-year results and cost analysis}

SZ $\operatorname{Tan}^{1,2,3}$ and $\mathrm{L} \mathrm{Au^{2 }}$

\begin{abstract}
Purpose To evaluate the safety, efficacy, and the cost of combined phacoemulsification and single iStent insertion in open angle glaucoma (OAG) at 3-years follow-up. Methods This was a prospective, uncontrolled, interventional case series. All subjects underwent single iStent implantation combined with cataract surgery by a single surgeon and were followed up over 3 years. Primary outcome measures were the reduction in intraocular pressure (IOP) and number of glaucoma drops at 1, 2, and 3 years. The costs of the procedure vs the cost of continuation of glaucoma drops were calculated and compared in patients who completed 3-years follow-up.

Results Forty-one patients were included in the study and thirty-six patients completed 3-years follow-up. Mean pre-op IOP was $21.2 \mathrm{~mm} \mathrm{Hg}$ on 2.1 medications. Mean IOP was reduced to $15.9 \mathrm{~mm} \mathrm{Hg}$ on 0.5 drops, $16.1 \mathrm{~mm} \mathrm{Hg}$ on 1.0 drops, and $17.1 \mathrm{~mm} \mathrm{Hg}$ $(P<0.001)$ on 1.3 drops $(P<0.001)$ at 1,2 , and 3 years, respectively. The overall cost of combined cataract surgery and iStent was estimated to be $£ 829.32$ more in total than conservative management with brand name eye drops over 3 years $(£ 7.70$ per patient per year) and $£ 14176.9$ more if generic drops were used. ( $£ 131.3$ per patient per year). Conclusions Combined phaco-iStent proved to be a safe and effective way of managing patients with OAG over our 3-year follow-up period. The cost-effectiveness of the procedure may vary depending on whether brand name or generic eye drops are used.

Eye (2016) 30, 1365-1370; doi:10.1038/eye.2016.139; published online 8 July 2016
\end{abstract}

\section{Introduction}

Glaucoma remains one of the commonest causes of blindness in the world. Treatment of suboptimal intraocular pressure (IOP) can be with topical eye drops, laser therapy, and surgery. Topical eye drops require compliance from patients and are not always well-tolerated. Laser therapy such as Selective Laser Trabeculoplasty (SLT) and Argon Laser Trabeculoplasty (ALT) can lower IOP variably but tend not to have a long-lasting effect. Gold standard glaucoma surgery including trabeculectomy and tube surgeries lower IOP effectively, but have higher complication rates and require intensive post-operative care. In recent years, a new class of procedures, termed micro-invasive glaucoma surgery (MIGS) is gaining popularity, offering an alternative and safer way to lower IOP and reduce the burden of medications. iStent (Glaukos Corporation, Laguna Hills, CA, USA), one of first and most commonly used MIGS devices, is a heparincoated, non-ferromagnetic, titanium stent $1 \mathrm{~mm}$ in length and $0.3 \mathrm{~mm}$ in height. The stent is designed to cannulate Schlemm's canal and connect this space directly to the anterior chamber, allowing aqueous humour to egress into Schlemm's canal more efficiently, thereby lowering IOP.

The Manchester iStent study by Patel et al showed that combined iStent and cataract surgery lowered the mean IOP from $21.1 \mathrm{~mm} \mathrm{Hg}$ at baseline to $16.7 \mathrm{~mm} \mathrm{Hg}$ at 6 months $(P<0.01)$. The mean number of drops was also reduced from 2.3 to $0.6(P<0.01)$ with $66 \%$ of patients being drop-free at 6 months.

This article reports the 3-year results of the Manchester iStent study. The authors also performed cost analysis in this cohort of patients.

\section{Materials and methods}

This study was a single-surgeon, single-centred, uncontrolled, prospective interventional case series. Forty-one patients who had ab interno single iStent implantation combined with cataract surgery were included in this study. Inclusion criteria were mild or moderate glaucoma (primary, angle recession, pseudoexfoliation, previous narrow angle with
${ }^{1}$ Centre for Ophthalmology and Vision Sciences, Institute of Human Development, Faculty of Medical and Human Sciences, University of Manchester, Manchester, UK

${ }^{2}$ Manchester Royal Eye Hospital, Central Manchester University Hospitals NHS Foundation Trust, Manchester, UK

${ }^{3}$ Centre for Advanced Discovery and Experimental Therapeutics (CADET), Central Manchester University Hospitals NHS Foundation Trust, Manchester Academic Health Sciences Centre, Manchester, UK

Correspondence:

L Au, Department of Ophthalmology, Manchester Royal Eye Hospital, Oxford Road, Manchester M13 9WL, Lancashire, UK Tel: +44 (0)161 276 5522; Fax: +44 (0)1612765986. E-mail: Leon.Au@cmft.nhs.uk

Received: 5 August 2015 Accepted in revised form: 18 April 2016 Published online: 8 July 2016 
current open angle following peripheral iridotomy), current use of at least one glaucoma medication and a visible scleral spur with gonioscopy. Exclusion criteria were other types of glaucoma, history of any glaucoma surgery or cyclodestructive procedure, elevated episcleral venous pressure, and peripheral anterior synechiae in the nasal angle. Informed consent was obtained from all patients by qualified ophthalmologists after thorough explanation of the risks and benefits of the procedure. All procedures followed the tenets of the Declaration of Helsinki. We certify that all applicable institutional and governmental regulations concerning the ethical use of human volunteers were followed.

The surgical procedure has been previously reported. ${ }^{1}$ All patients were advised to discontinue the use of all glaucoma medications following surgery. Post-operative drops were topical chloramphenicol qds and dexamethasone $0.1 \%$ qds for three weeks. Patients were reviewed at 1 week, 1 month, 3 months, and 6 months, where the best-corrected visual acuity (BCVA), IOP, and number of drops were recorded using a pre-designed proforma. Subsequent follow-ups were determined by the principal investigator based on clinical needs. Number of glaucoma drops and IOP were recorded at 1-year, 2-year, and 3-year follow-up visits. Hypotensive treatment was recommenced at the discretion of the principal investigator, taking into account the disease progression and IOP.

The primary outcome measures were the reduction in IOP and number of glaucoma drops at 1 year, 2 years, and 3 years.

The costs of the procedure $v s$ the cost of continuation of glaucoma drops were also compared. We recorded the actual drops patients were prescribed pre-operatively, at 1 year, 2 years, and 3 -years post-operatively. Two separate cost-analyses were performed: one based on the use of brand name drops and the other on generic drops. The cost for brand name drops was obtained from the British National Formulary (BNF) March 2010 (period when the patients were recruited) and the cost of generic drops were obtained from the latest online BNF (February 2016) to reflect current cost. Where more than one generic drops is available, the cheapest was used for the purpose of this analysis. Among the commonly prescribed drops in our trust during the study period were the prostaglandin analogues (Lumigan, Xalatan, Travatan, Saflutan), B-blockers (Timolol 0.25\%, Timolol LA, Tiopex), carbonic anhydrase inhibitors (Azopt, Trusopt, Trusopt preservative free), alpha-agonists (Alphagan, Iopidine $0.5 \%$, Iopidine $1 \%$ ), and the combination drops (Cosopt, Cosopt preservative free, Ganfort). Only the B-blockers were available in generic form during the study period. Generic drops available at the end of the study period were Latanoprost, Brinzolamide, Dorzolamide, Brimonidine, Dorzolamide/Timolol, and Dorzolamide/Timolol unit dose. All glaucoma drops used in our unit have an opening shelf life of 28 days and the number of bottles required per year per patient is 13.03 bottles (365/28 days). A wastage adjustment was also applied to account for misadministration of medication by patients and non-compliance.

The adjustment factor was determined from a previous retrospective study that examined the prescription refill frequency of 27000 patients; this estimate increased our theoretical annual cost by $21 \% .^{2}$ Only patients who completed 3-years follow-up were used in the cost analysis.

The surgical cost included the cost of the iStent and the cost of disposable instruments, and viscoelastic materials required to perform the surgery, and excluded the cost of reusable instruments, surgeon's time, and theatre time, as these were considered to be fixed cost in the National Health Service in the United Kingdom (UK), irrespective of whether iStent surgery was being performed. One iStent (Glaukos Corporation) costs $£ 480$ in the UK. The cost of a routine phacoemulsification and intraocular lens (IOL) implant including all disposable instruments, IOL (Akreos Adapt AO, Bausch+Lomb, USA), one Healon OVD (Abbott Medical Optics, USA), and one Healon GV OVD (Abbott Medical Optics, USA) amounted to $£ 180.72$. Total cost for the combined surgical procedure was therefore $£ 660.72$ ( $£ 480+180.72)$.

Statistical analysis was performed using Microsoft Excel. Statistical significance was taken as $P<0.05$.

\section{Results}

The mean age of the patients was $76.1 \pm 10.38(\mathrm{SD})$ years old, with a female to male ratio of 1.4:1. Out of the 41 patients recruited for this study, $3(7.3 \%)$ patients had pseudoexfoliation, 2 (4.9\%) had angle recession, 4 (9.8\%) had normal tension glaucoma, 2 (4.9\%) had previous narrow angle glaucoma, and the rest (30 patients, 73.1\%) had primary open angle glaucoma. (Table 1) Nine patients had previous SLT or ALT, and two had peripheral iridotomies. The mean cup-disc ratio and mean deviation on Humphrey visual field assessment were 0.7 and $-9.08 \mathrm{DB}$, respectively. Thirty-six (88\%) patients completed 3-years follow-up. Out of the five patients who dropped out of the study, two (5\%) went on to have trabeculectomy after 2 years because of suboptimal IOP control; one was referred back to a

Table 1 Glaucoma subtypes in the study patients

\begin{tabular}{lr}
\hline Type of glaucoma & $n(\%)$ \\
\hline Pseudoexfoliation & $3(7.3)$ \\
Angle recession & $2(4.9)$ \\
Normal tension & $4(9.8)$ \\
Previous narrow angle & $2(4.9)$ \\
Primary open angle & $30(73.1)$ \\
\hline
\end{tabular}


district general hospital for follow-up; and two failed to attend follow-up appointments after 2 years.

The mean pre-operative BCVA was $0.52 \pm 0.28 \log \mathrm{MAR}$, and it improved to $0.25 \pm 0.27 \log \mathrm{MAR}$ at 1 month and was maintained at $0.23 \pm 0.31 \log \mathrm{MAR}$ at 6 months postoperatively $(P=0.003)$. The mean pre-operative IOP was $21.2 \pm 4.7 \mathrm{~mm} \mathrm{Hg}$ on $2.1 \pm 1.0$ medications, with six $(14.6 \%)$ patients on acetazolamide. At 1 week post-operation, mean IOP was $19.8 \pm 7.0 \mathrm{~mm} \mathrm{Hg}$ on no drops and no acetazolamide; $19.8 \pm 5.8 \mathrm{~mm} \mathrm{Hg}$ on $0.2 \pm 0.5$ drops at 1 month; $16.3 \pm 2.9 \mathrm{~mm} \mathrm{Hg}$ on $0.5 \pm 0.8$ drops at 3 months; and $16.1 \pm 3.4 \mathrm{~mm} \mathrm{Hg}$ on $0.6 \pm 0.9$ drops at 6 months. At 1-year post-op, mean IOP was reduced to $15.9 \pm 2.6 \mathrm{~mm} \mathrm{Hg}$ on $0.5 \pm 0.8$ drops; $16.1 \pm 2.9 \mathrm{~mm} \mathrm{Hg}$ on $1.0 \pm 1.1$ drops at 2 years; and $17.1 \pm 2.4 \mathrm{~mm} \mathrm{Hg}(P<0.001)$ on $1.3 \pm 1.2$ drops $(P<0.001)$ at 3 years (Figures 1 and 2$)$. Sixty-three per cent of patients were on no medication at 1-year, 50\% at 2-years, and $32 \%$ at 3 -years post-operation (Figure 1).

Transient hyphaema occurred in one patient and resolved within 1 week. All patients had either improvement in vision or stable vision at 6 months-follow-up.

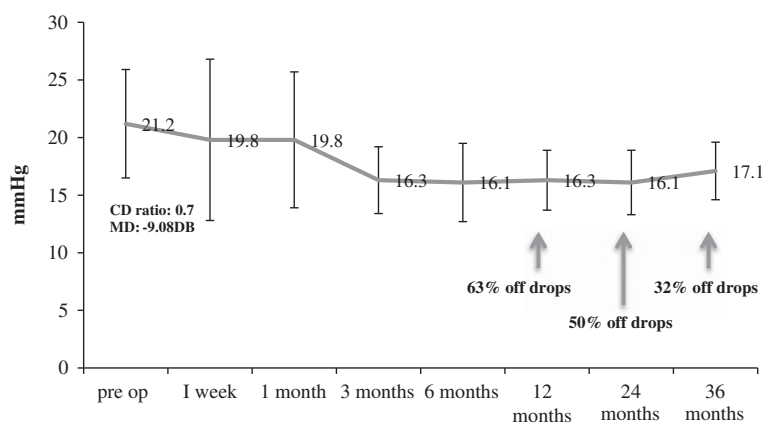

tim

Figure 1 Mean IOP (+ / - standard deviation) over 3-year followup period with percentage of patients off hypotensive drops. Pre-op mean cup-disc ratio was 0.7 with visual field mean deviation of $-9.08 \mathrm{DB}$.

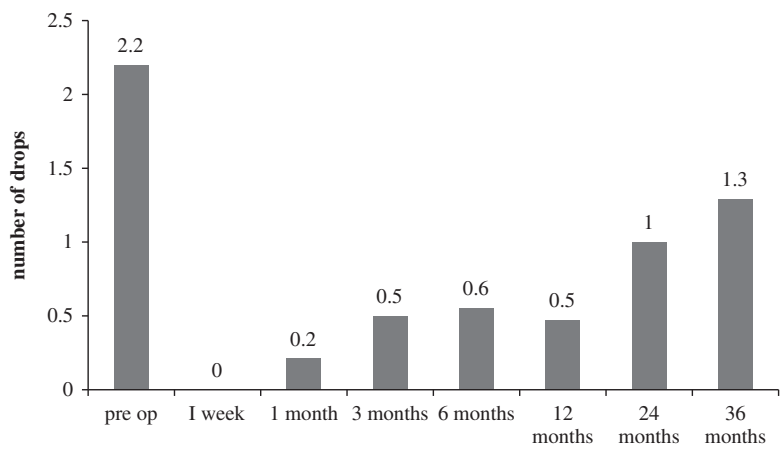

time

Figure 2 Mean number of hypotensive drops over 3-year follow-up period.

\section{Cost analysis}

Cost analysis was performed in 36 patients who completed 3-years follow-up. If the patients did not undergo surgery and were kept on the same regime for the 3-year period, the 'theoretical' total cost of drops would be $£ 35,768.70$ for brand name drops and $£ 17716.2$ for generic drops. The 'actual' cost of glaucoma brand name drops for this cohort during the study period was $£ 1866.8, £ 4440.0$ and $£ 6505.3$ at 1 -year, 2-years, and 3-years post-surgery, respectively, giving a total cost of $£ 12812$.10 over a 3-year period; generic drop cost was $£ 1250.6, £ 2656.9, £ 4199.7$ at each year, respectively (total cost $=£ 8107.2)$. The combined cost of treatment for 36 patients amounted to $£ 36,598.02$ for brand name drops (surgical cost, $£ 23785.92(£ 660.72 \times 36)+$ actual cost of drops, $£ 12812.10)$ and $£ 31893.12$ if generic drops were used. Overall, combined cataract surgery and iStent cost $£ 829.32$ ( $£ 36598.02$ - $£ 35768.7$ ) more than conservative management with brand name eye drops ( $£ 7.70$ per patient per year) and $£ 14,176.9$ more if generic drops were used. ( $£ 131.3$ per patient per year). Figure 3 compares the cumulative costs of combined phaco-iStent vs conservative management with eye drops for both brand name drops and generic drops.

\section{Discussion}

MIGS such as iStent has increased in popularity recently. It can be performed as a single procedure or combined with cataract surgery. Although cataract surgery alone is known to reduce the IOP, a recent meta-analysis showed that combining the procedures have the added benefit of greater reduction in IOP and the number of antiglaucoma drops required. ${ }^{3}$ When iStent alone was implanted, the magnitude of IOP lowering was found to match studies with combined procedure in one case series. ${ }^{4}$ Vandewalle et $a l^{5}$ compared iStent alone vs combined phaco-iStent and found a greater benefit with the combined procedures. All our patients in this study had some degree of cataract and hence combined procedure was performed.

Our study demonstrated that single iStent implantation when combined with cataract surgery not only significantly improved BCVA by more than two lines but also reduced IOP and the number of hypotensive drops significantly from 1 week post-operatively, as previously described in the early results of the Manchester iStent study. ${ }^{1}$ At 1 year, the mean IOP was reduced by $5.3 \mathrm{~mm} \mathrm{Hg}(25 \%)$ compared with baseline and this was achieved with 1.6 fewer drops. These results were comparable with the two randomised controlled trials $(\mathrm{RCT})^{6,7}$ that showed a reduction of IOP of $17 \%$ and $33 \%$ with 1.6 and 1.4 drop reduction, respectively, at 1-year follow-up. Our 2-years follow-up 


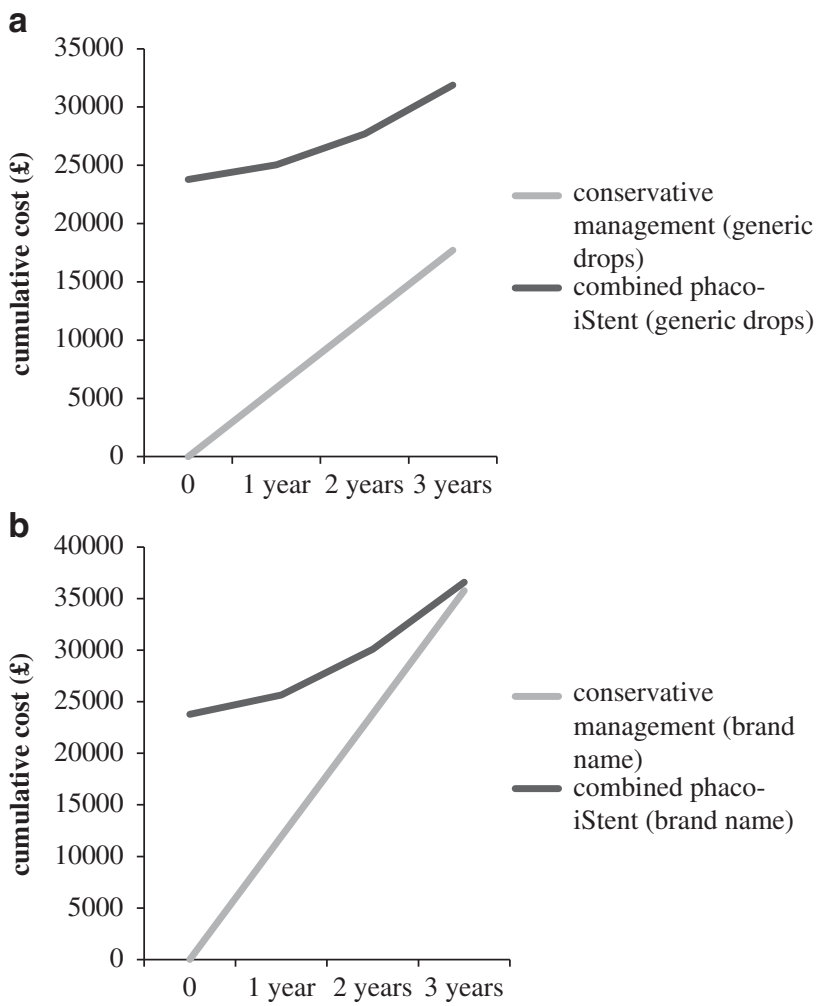

Figure 3 Cumulative costs of combined phaco-iStent $v$ s conservative management with glaucoma drops for both generic drops (a) and brand name drops (b).

results showed a $24 \%$ IOP reduction with 1.1 drop reduction, compared to $33 \%$ IOP reduction and 1.3 drop reduction in the RCT conducted by Craven et al ${ }^{8}$ At 3 years, IOP was reduced by $4 \mathrm{~mm} \mathrm{Hg}$ (19\%) on 0.8 fewer hypotensive eye drops compared to pre-op in our study group. The longest reported follow-up period in the literature to date was 5 years in a small prospective case series $(n=19)$ by Arriola-Villalobos et al ${ }^{9}$ The group showed a reduction of IOP by $3 \mathrm{~mm} \mathrm{Hg}(16 \%)$ with a drop reduction of 0.5 at the end of a mean follow-up period of 54 months.

Only one subject developed complication in the form of hyphaema in our study. Transient hyphaema is a recognised complication within the first post-operative week as reported in a few case series. ${ }^{4,10}$ Among other adverse events reported in studies are stent malposition or occlusion early in the post-operative period, affecting $4 \%$ to $18 \%$ of cases but usually resolved with observation alone or secondary procedures such as Nd:YAG laser, argon laser gonioplasty, recombinant tissue plasminogen activator, stent repositioning, or stent replacement. ${ }^{10,11}$ Steroid response was found in 2 of 53 eyes 4 weeks post-operatively in a case series where IOP was controlled after cessation of steroids. ${ }^{10}$ This was also the experience of Le et $\mathrm{al}^{12}$ who recommended early tapering of steroids. Steroid response may partly explain the delay in the peak
IOP lowering effect following iStent insertion, usually at 3 months post-insertion, 7,11 which was also what we found in our study. There was no long-term adverse event in the 3-year follow-up period in our study group, suggesting that the procedure is a safe and effective longterm method of lowering IOP. Two patients in our cohort required trabeculectomy $(5 \%)$ at 2-years follow-up. One of the patients had severe ocular surface disease and drop intolerance requiring acetazolamide pre-operatively to achieve an IOP of $24 \mathrm{~mm} \mathrm{Hg}$; the other patient had moderate glaucoma requiring three eye drops pre-operatively to achieve an IOP of $20 \mathrm{~mm} \mathrm{Hg}$. The rate is comparable to that reported in the landmark RCT where around $2 \%$ of the study subjects progressed to require trabeculectomy. 7,8

The cost analysis showed that at $£ 7.70$ per patient per year, our cohort of patients had a lower mean IOP at $17.1 \mathrm{~mm} \mathrm{Hg}$ compared to $21.2 \mathrm{~mm} \mathrm{Hg}$ pre-operatively and a 3-line improvement in visual acuity in a 3-year follow-up period. In addition, there may also be improvement in quality of life with fewer numbers of topical drops and improved vision, although research into quality of life assessment is still required. The cost analysis was performed taking into account the cost of cataract surgery. In real life, most of the patients in this cohort would have required cataract surgery at some stage in the 3-year follow-up period with or without the iStents to improve vision, and 
combining the two procedures have the added advantage of saving surgeons' and theatre time, which averages to cost $£ 1200$ per hour. ${ }^{13}$ Hence, the actual cost saved in combined phaco-iStent procedure vs topical medications could be higher than that estimated here. In this study, we also looked at the cost if the drops were dispensed as generic. Unsurprisingly, it is 16-fold cheaper ( $£ 131.30$ more per patient per year) compared with brand name drops, making combined phaco-iStent less attractive from the cost point of view in countries where generic drops are commonly prescribed. However, generic drops are not without issues. There have been studies to suggest that generic latanoprost is less tolerated by patients compared with Xalatan (Pfizer Inc., New York, NY, USA) drops. ${ }^{14,15}$ Other reported issues with generic latanoprost included failure of a bottle to last a month, difficulty opening the cap and the lack of compliance aid. ${ }^{14}$ There is also ongoing debate about the efficacy of generic vs brand name drops. These issues will ultimately have an impact on clinician's time, patient management, patient satisfaction, and the cost of glaucoma care.

To the best of our knowledge, this is the first cost analysis study that looked at the actual cost of combined surgery $v$ s topical glaucoma drops in a cohort of clinical patients treated under the National Health System. Iordanous et al ${ }^{16}$ looked at the 6 years projected cost of iStents alone without cataract surgery vs topical medications in Canada and found a cumulative cost difference of $\$ 20.77, \$ 1272.55$, and $\$ 2124.71$ per patient when comparing iStent $v$ s monodrug, bidrug, and tridrug therapy, respectively. The authors employed a slightly different cost analysis method to ours whereby the overall cost was estimated by taking a weighted average of the cost of each class of glaucoma medications instead of the actual cost of drops for each individual patient. Our study looked into the costs of topical medication vs surgery, but did not take into account the indirect costs of surgery (theatre time, surgeon's time, non-disposable instruments), follow-up visits, side effects of treatment, and quality of life assessment. These parameters are difficult to assess, in the same way that if our cohort were not to undergo surgery how many more follow-up visits would they require and what proportions of them would require filtration surgery. Further studies are therefore required to establish the cost-effectiveness of iStent and its role in the glaucoma treatment paradigm.

Complete success in glaucoma treatment can be defined by $\mathrm{IOP} \leq 21 \mathrm{~mm} \mathrm{Hg}$ without medications. ${ }^{17,18}$ In our study, $63 \%, 50 \%$, and $32 \%$ fulfilled this criterion at 1 year, 2 years, and 3 years, respectively. There seems to be a trend of increasing IOP and number of hypotensive drops with time from the insertion of the iStents. The procedure is by no means a long-term permanent solution in highrisk patients with advanced glaucoma, but our study showed that it is a safe and potentially cost-effective way (especially in countries where brand name drops are used) of managing patients who have mild to moderate glaucoma and cataracts. It also obviates the issues of non-adherence and side effects from topical medications. The study was limited by the small number of patients, and hence further larger prospective study with longer follow-up period is required to establish the long-term effectiveness of iStent in the management of glaucoma.

\section{Summary}

\section{What was known before}

- iStent lowers intraocular pressures in open angle glaucoma.

\section{What this study adds \\ - Three-year outcome data of iStents. \\ - Cost analysis of this intervention.}

\section{Conflict of interest}

The authors declare no conflict of interest.

\section{References}

1 Patel I, de Klerk TA, Au L. Manchester iStent study: early results from a prospective UK case series. Clin Experiment Ophthalmol 2013; 41(7): 648-652.

2 Platt R, Reardon G, Mozaffari E. Observed time between prescription refills for newer ocular hypotensive agents: the effect of bottle size. Am J Ophthalmol 2004; 137(1 Suppl): S17-S23.

3 Malvankar-Mehta MS, Iordanous Y, Chen YN, Wang WW, Patel SS, Costella J et al. iStent with phacoemulsification versus phacoemulsification alone for patients with glaucoma and cataract: a meta-analysis. PLoS One 2015; 10(7): e0131770.

4 Buchacra O, Duch S, Milla E, Stirbu O. One-year analysis of the iStent trabecular microbypass in secondary glaucoma. Clin Ophthalmol 2011; 5: 321-326.

5 Vandewalle E, Zeyen T, Stalmans I. The iStent trabecular micro-bypass stent: a case series. Bull Soc Belge Ophtalmol 2009; 311: 23-29.

6 Fea AM. Phacoemulsification versus phacoemulsification with micro-bypass stent implantation in primary open-angle glaucoma: randomized double-masked clinical trial. J Cataract Refract Surg 2010; 36(3): 407-412.

7 Samuelson TW, Katz LJ, Wells JM, Duh YJ, Giamporcaro JE, Group UiS. Randomized evaluation of the trabecular microbypass stent with phacoemulsification in patients with glaucoma and cataract. Ophthalmology 2011; 118(3): 459-467.

8 Craven ER, Katz LJ, Wells JM, Giamporcaro JE, Group iS. Cataract surgery with trabecular micro-bypass stent implantation in patients with mild-to-moderate open-angle glaucoma and cataract: two-year follow-up. J Cataract Refract Surg 2012; 38(8): 1339-1345. 
9 Arriola-Villalobos P, Martínez-de-la-Casa JM, Díaz-Valle D, Fernández-Pérez C, García-Sánchez J, García-Feijoó J.

Combined iStent trabecular micro-bypass stent implantation and phacoemulsification for coexistent open-angle glaucoma and cataract: a long-term study. Br J Ophthalmol 2012; 96(5): 645-649.

10 Belovay GW, Naqi A, Chan BJ, Rateb M, Ahmed II. Using multiple trabecular micro-bypass stents in cataract patients to treat open-angle glaucoma. J Cataract Refract Surg 2012; 38 (11): 1911-1917.

11 Spiegel D, García-Feijoó J, García-Sánchez J, Lamielle H. Coexistent primary open-angle glaucoma and cataract: preliminary analysis of treatment by cataract surgery and the iStent trabecular micro-bypass stent. Adv Ther 2008; 25(5): 453-464.

12 Le K, Saheb H. iStent trabecular micro-bypass stent for open-angle glaucoma. Clin Ophthalmol 2014; 8: 1937-1945.

13 Improving quality and efficiency in the operating theatre. A lifeline for financial leaders: Institute for Innovation and Improvement; 2009. Available at http://harmfreecare.org/
wp-content/files_mf/Improving-quality-and-efficiency-inthe-operating-theatre.pdf.

14 Painter SL, Mead AL. Patient experience of the transition from Xalatan to generic latanoprost. Eye 2014; 28(7): 911.

15 Takada Y, Okada Y, Fujita N, Saika S. A patient with corneal epithelial disorder that developed after administration of a latanoprost generic, but not a brand-name drug, eye drop. Case Rep Ophthalmol Med 2012; 2012: 536746.

16 Iordanous Y, Kent JS, Hutnik CM, Malvankar-Mehta MS. Projected cost comparison of Trabectome, iStent, and endoscopic cyclophotocoagulation versus glaucoma medication in the Ontario Health Insurance Plan. J Glaucoma 2014; 23(2): e112-e118.

17 Singh K, Lee BL, Wilson MR, Group GMR-LM. A panel assessment of glaucoma management: modification of existing RAND-like methodology for consensus in ophthalmology. Part II: Results and interpretation. Am J Ophthalmol 2008; 145: 575-581.

18 Shaarawy T, Sherwood M, Grehn F. Guidelines on Design and Reporting of Glaucoma Surgical Trials. Kugler publications: Amsterdam, The Netherlands, 2009. 